\title{
Editorial
}

\section{High Content Screening for Lead Identification and Optimization}

\section{Zhuyin $\mathrm{Li}^{*}$}

National Center for Advancing Translational Sciences, National Institutes of Health, 9800 Medical Center Drive, MSC 3370, Bethesda, MD 20892, USA

Automated microscope-based High Content Screening (HCS or HCA) has gained tremendous momentum recently because of its ability to capture many subcellular features simultaneously in complex biology systems, and/or to monitor cellular processes that are otherwise intractable using conventional technologies. HCS can be utilized in early drug discovery and preclinical development to accelerate drug discovery. It has been widely used in target validation, lead generation, toxicity studies, and drug mechanism studies. HCS also has the potential to be used to support clinical trials, such as companion diagnostics.

In this special issue on HCS, three HCS-based assays that are applicable for lead identification and optimization are highlighted. To many institutions, the initiation of primary screening is a serious commitment to the targets, pathways or disease hypothesis. Therefore, patho-physiological relevancy, cost, throughput, scalability, quality, etc. must be carefully weighted when designing an assay for primary screening. Unlike traditional HTS, which has one or two measurements, HCS enables one to measure many parameters or features of individual cells or organisms simultaneously. With currently available technologies, HCS-based HTS is still labor-intensive and could be very costly if multiple dyes and/or antibodies are used. Therefore, HCS-based HTS should be considered only if there are no adequate conventional screening technologies that could be used to obtain similar information. In this issue, Peppard and colleagues present an HCS-based HTS entitled "Identifying small molecules which inhibit autophagy: a phenotypic screen using image-based high-content cell analysis" [1] to identify smallmolecule autophagy inhibitors by following the cytoplasmic redistribution of GFP tagged LC3, a component of the autophagosome, from diffuse to punctate dots in HeLa cells.

Overexpression of tagged proteins may interrupt the interaction between the protein and its partners in the cellular network, thus leading to non-physiologically relevant phenotypes. Therefore, many use antibody stains to monitor the translocation and expression of target proteins, or changes of

*Address correspondence to this author at the National Center for Advancing Translational Sciences, National Institutes of Health, 9800 Medical Center Drive, MCS 3370, Bethesda, MD 20892, USA; Tel: 301-217-5727; Fax: 301-217-5736; E-mail: zhuyin.li@nih.gov organelle and cell structures and functions. Antibodies in general are expensive and the use of multiple antibody stains in primary screening could be cost prohibitive, and the multiple wash steps maybe not HTS friendly. However, antibody staining-based HCS assays have played and will continue to play key roles in secondary and tertiary assays, as well as mechanism and toxicity studies. Sum and his colleagues describe the development of an antibody stain-based HCS for microtubule structure, entitled "Establishing a High-Content Analysis Method for Tubulin Polymerization to Evaluate Both the Stabilizing and Destabilizing Activities of Compounds" [2]. This assay enables the understanding of the mechanisms of action for tubulin-interacting compounds.

Two-dimensional (2-D) monolayer culture cells are routinely employed in primary HTS and in lead generation and optimization. However, these 2-D model systems do not recapitulate the complexity of the 3-D organization and the pathophysiology in tissues and organs. Therefore, the practice of using 2-D cellular models for drug testing is being questioned for its inability to mimic physiological environments of diseased cells in the human body. An increased number of laboratories has started to establish 3-D cellular models for drug testing. Although much less complex than in vivo models or complete organisms, these 3 -D cellular models are one-step closer to physiological conditions. In the manuscript of "Live Multicellular Tumor Spheroid Models For High-Content Imaging and Screening In Cancer Drug Discovery" [3], Reid and his colleagues report the development of a multicellular tumor spheroid (MCTS) model for drug screening and a simple yet predictive 3-D image analysis algorithm to quantify changes induced by drugs.

In summary, due to its flexibility and information-rich nature, HCS has become the choice for many scientists to examine the temporal and spatial effects of compounds on cells, not only for the intended targets, but also for other cellular targets and pathways. This feature not only enables the identification of the best hits and leads from screening, but also facilitates multi-parameter optimization of leads.

\section{REFERENCES}

[1] Peppard JV, Rugg C, Smicker M, Dureuil C, Ronan B, Flamand O, Durand L, and Pasquier B. Identifying small molecules which inhibit autophagy: a phenotypic screen using image-based highcontent cell analysis. Curr Chem Genom Transl Med 2014; 8: 3-15. 
[2] Sum CS, Nickischer D, Lei M, Weston A, Zhang L, Schweiser L. establishing a high-content analysis method for tubulin polymerization to evaluate both the stabilizing and destabilizing activities of compounds. Curr Chem Genom Transl Med 2014; 8; 16-26.
[3] Reid BG, Jerjian T, Patel P, Zhou Q, Yoo BH, Kabos P, Sartorius, $\mathrm{CA}$, and LaBarbera DV. live multicellular tumor spheroid models for high-contentimaging and screening in cancer drug discovery. Curr Chem Genom Transl Med. 2014; 8: 27-35

(C) Zhuyin Li; Licensee Bentham Open.

This is an open access article licensed under the terms of the Creative Commons Attribution Non-Commercial License (http://creativecommons.org/licenses/by-nc/3.0/) which permits unrestricted, non-commercial use, distribution and reproduction in any medium, provided the work is properly cited. 